\title{
ENVIRONMENT AND FINANCE: WHY WE SHOULD MAKE THE ENVIRONMENT A PART OF THE FINANCIAL MARKETS
}

\author{
Monique Jeanblanc
}

Université d'Evry Val d'Essonne

\author{
Wojciech Szatzschneider* \\ School of Actuarial Sciences, Universidad Anáhuac del Norte
}

(Received 6 november 2001, accepted 3 january 2002)

\begin{abstract}
This paper represents a proposal to create a kind of financial markets out of environmental improvements. We shall explain how to do it, and why the financial approach is practically the only one able to stop and invert the environmental degradation. Particularly, we shall concentrate our attention in deforestation and show how to produce effectively reforestation. With a model of the current and future situation, we consider a $0-t h$ dimensional squared Bessel process with drift. We justify our choice as a limit of branching phenomena. In the game of reforestation versus deforestation, we solve the optimization problem of the first move needed to start the project. On the other hand, because we are aiming for the creation of a developed market, we consider the classical Merton's problem with underlying asset instead of Geometric Brownian motion.
\end{abstract}

\section{Resumen}

Este trabajo representa una propuesta para crear una clase de mercados financieros de mejoras ambientales. Explicaremos cómo hacerlo, y por qué el enfoque financiero es prácticamente el único capaz de detener e invertir la degradación ambiental. Particularmente, concentramos nuestra atención en la deforestación y mostramos cómo reforestar con eficacia. Con un modelo de la situación real y futura, consideramos un proceso de Bessel de dimensión cero con tendencia. Justificamos esta elección como el límite de fenómenos de ramificación. En el juego de la reforestación, resolvemos el problema de optimización sobre el primer movimiento necesario para comenzar el proyecto. Por otra parte, ya que nuestro objetivo es la creación de un mercado desarrollado, consideramos el problema clásico de Merton con un activo subyacente en lugar de un movimiento geométrico Browniano.

JEL Classification: G1, and G13.

Keywords: Financial markets, Contingent pricing.

* Escuela de Actuaría, Universidad Anáhuac del Norte, Avenida Lomas Anáhuac s/n, Col. Lomas Anáhuac, Huixquilucan, 52786 Edo. de México, Telephone: (52)5627-0210, Fax:

(52)5596-1938, E-mail: wojciech@anahuac.mx.

The authors wish to thank two anonymous referees for comments. 


\section{Introduction}

Deforestation, loss of biodiversity and $\mathrm{CO}_{2}$ emissions are only some of many environmental problems that must be treated urgently. We will not present statistics, since they can be found all over. Our goal is to invert things, and commence with reforestation taking this word a little bit freely.

Reforestation is still by far one of the easiest to use words in the environmental language. Words like restoration and, particularly, "integrity" (emerging from ethics and landing in environmental science without sufficient justifications) make things very vague.

There is also a list of problems in the practicality of sustainable development. Here are some of the reasons:

1. The utopian vision about the possibility of improvements using the intrinsic value of nature without involving any money.

2. Decisions considering the environment are taken when possible results practically cannot be evaluated, and thus are inadequate, and on the other hand (as stated by global integrity project) to conserve integrity we must be guided by a knowledge of our actions consequences, for example see Crabbe et al. (2000), which is no more than a vicious circle.

3. Agents responsible for new projects often go around the law, which frequently is not precise enough.

4. The call for "back to nature" is pure nonsense, particularly if it is referred to undeveloped (read poor) countries.

5. Using governmental programs in undeveloped countries is once again wasting money. Drawing examples from India, where governmental programs are supposed to work (cf. Oates (1999)), and trying to apply them in say, Africa or Latin America, shows poignant lack of knowledge about differences between religions, cultures and regions.

6. An International Analog Forestry Project can offer only local and reduced solutions. We cannot expect that huge numbers of humans could make their lives "picking berries" (it is of course an oversimplification made on purpose by the authors of this study).

7. Environmentalist groups frequently posses only a dim interest.

We have reviewed some topics from the environment just to show that our program probably is the only one that could work. But, before explaining why it could be done, we still need one more comment. Nowadays, environmentalists blame Bush's presidency because of its anti-environmentalism. This simply shows that their work has been very fragile. Our project is based on "positive action awards", and there is a need for funds, either public or coming from environmental organizations. Many resources are frequently wasted because of:

1. Extremely expensive consulting. 
2. Failed environmental projects frequently supporting corrupt agents involved.

During the last years, we have observed the development of modern finance in fields related to our idea. Firstly, the creation of derivatives products, where the underlying asset cannot be directly priced such as:

\section{Real Options.}

2. Options on climate and others.

Secondly, there have been many recent developments on market frictions such as: transaction costs, asymmetries, or insiders (agents with additional knowledge). All these allow for the formulation and solution of new challenging problems. We will now look into the current environmental programs that are related to finance. In our opinion, the most important ones are:

1. Financial institutions have made a commitment that they would not support new projects if the environment is at stake (hard to analyze).

2. Insurance companies reduce premiums to cleaner industries.

3. Permits to pollute in micro or macro scale.

The first two topics are agreements between parties and can be distorted. As stated by Merton and Bodie, we pretend for the environment to become a part of the financial innovation spiral. Among other benefits it would provide transparent ways to transfer economic resources. Recently, popular third project finds many difficulties to handle problems. Our approach, with a different underlying model, could be applied more directly to any form of pollution.

The paper is organized as follows. In the next section, we work out a model before the financial intervention. In section 3, we deal with financial intervention. In section 4 , we make several comments about financial markets on environment. In section 5, we study Merton's problem. In section 6, we establish further valuation topics. Finally, in section 7 , we present conclusions.

\section{The Model before the Financial Intervention}

To model the number of trees in a given region, we propose a 0 - $t h$ dimensional squared Bessel process, $X(t), t \geq 0$, with negative drift defined by:

$$
\mathrm{d} X(t)=2 \sqrt{\sigma X(t)} \mathrm{d} W(t)+2 \beta X(t) \mathrm{d} t,
$$

where $X(0)=x_{0}>0$, and $\beta<0$. This process can also be viewed as a time changed BESQ (Bessel squared process). More precisely, if $\sigma=1$,

$$
X(t)=e^{2 \beta t} R\left[\frac{1-e^{-2 \beta t}}{2 \beta}\right],
$$


where $R$ is a BESQ with dimension 0 (or index -1 ).

In the sequel, for the sake of simplicity, we will set $\sigma=1$. The general case follows by a multiplication by $\sigma$. This is, of course, also 0-th dimensional Cox, Ingersoll, and Ross model for interest rates. $X(\cdot)$ will eventually become zero before $t$, and, if so, it will stay there forever. If not, the remaining probability has the density:

$$
f_{(x, y)}(t)=\frac{\beta}{2 \sinh (\beta t)}\left(\frac{x}{y}\right)^{\frac{1}{2}} \exp \left\{-\beta \frac{x \exp (\beta t)+y \exp (-\beta t)}{2 \sinh (\beta t)}\right\} I_{1}\left(\frac{\beta \sqrt{x y}}{\sinh (\beta t)}\right),
$$

where

$$
I_{1}(z)=\frac{z}{2} \sum \frac{(z / 2)^{2 n}}{n !(n+1) !}
$$

In this study we will not do any statistics and will justify the model by a "heavy traffic approximation" instead. This can be done in many ways. For example, as an initial model we choose:

(i). The corresponding piecewise deterministic Markov process (PDMP) ( $c f$. Davis, 1984).

(ii). The Bachelier model, where the drift and volatility is changing properly.

Consider the PDMP process $U(t)$ with the infinitesimal generator:

$$
\mathcal{G} f(x)=\lambda x\left\{-(1+\theta) p_{1} f^{\prime}(x)-\int_{-\infty}^{\infty}[f(x-y)-f(x)] P(\mathrm{~d} y)\right\}
$$

The process $U(t)$ can be described heuristically as follows: $c U(t)$ is the rate of continous deforestation, where $c=-\lambda(1+\theta) p_{1}<0$ and the process moves in the deterministic way. This part is similar to insurance model in economic environment (the surplus gain interests) considered for example by Schmidli (1992). Further, with the rate $\lambda x$, where $x$ is the true actual number of trees, occurs reforestation (or deforestation) $X_{i}$, with probability law $P, X_{i}$ are i.i.d.r.v. (independently and identically distributed random variables) with $\mathrm{E}\left[X_{i}\right]=p_{1}$. Usually, and for many reasons, it is easier to reforestate in less devastated areas.

Suppose that the law $P$ has finite second moment $p_{2}$, and change $\lambda \rightarrow \lambda n$, $X_{i} \rightarrow X_{i} / \sqrt{n}, \theta \rightarrow \theta / \sqrt{n}$ (heavy traffic approximation). Then, we have:

\section{Proposition 1.}

$$
X_{n}(t) \Longrightarrow X(t), \quad \text { as } n \rightarrow \infty,
$$

with

$$
\sigma=\frac{\lambda p_{2}}{4}
$$


and

$$
\beta=\frac{-\lambda p_{1} \theta}{2}
$$

Proof:

We will give a sketch of the proof. It is enough to prove that, as $n \rightarrow \infty$ :

$$
\mathcal{G}_{n}(f(x)) \rightarrow \frac{1}{2} 4 \sigma x f^{\prime \prime}(x)+2 \beta x f^{\prime}(x)
$$

for any function $f \in C_{k}^{\infty}$ (with bounded support) ( $c f$. Ethier and Kurtz, 1986). We may assume that $|y|<c \sqrt{n}$ for some $c$. Using Taylor expansion results (1) in a straightforward way except, maybe, the fact that, as $n \rightarrow \infty$ :

$$
\frac{1}{\sqrt{n}} \int_{0}^{c \sqrt{n}}|y|^{3} P(\mathrm{~d} y) \rightarrow 0
$$

But this fact results from a kind of Kronecker's lemma since

$$
\frac{1}{k} \int_{0}^{c k}\left|y^{3}\right| P(\mathrm{~d} y) \rightarrow 0
$$

because

$$
\int_{-\infty}^{\infty} y^{2} P(\mathrm{~d} y)<\infty
$$

If we change signs in the infinitesimal generator $\mathcal{G}$, and drop " $x$ ", then the resulting process becomes classical Lundberg surplus model in risk theory. In this case, the well known result is that the heavy traffic approximation leads to:

$$
Y(t)=y_{0}+\sqrt{\lambda p_{2}} W(t)-\theta \lambda p_{1} t
$$

which is the classical Bachelier model. Making properly $\lambda$ state dependent one can easily obtain the same result as before. Notice that diffusion approximation in proposition (1) allows for a smoother analysis of problems, but the original process $U(t)$, perhaps with different jump part, could model better real situations.

\section{Financial Intervention}

Consider the "option" that pays:

$$
\int_{0}^{1}(X(s) \wedge k) \mathrm{d} s=k-\int_{0}^{1}\left(k-X_{s}\right)_{+} \mathrm{d} s
$$

For simplicity, we assume zero interest rate. Here $k$ is the maximum capacity in a given area. We will call it call option or "good" option (the payment is larger if more trees). The owner of the option paying the premium $c$, will possibly 
make some effort to obtain larger payment. That is, planting trees and protect them, if his investment will produce larger payments considering all expenses.

Consider $N$ options sold, and all holders acting in an optimal way, which now will be explained. The cost of planting (it is worth to do it at the beginning) is easy to set, and is $C_{0}$ per each tree, and protection would change the parameter $\beta \rightarrow B, B>\beta$ in our state variable. In this case, $B$ can be positive. This protection has the continuous cost $\delta=\delta(\beta, B, X(t))$. This part is difficult to set without empirical stridies, but here, we assume that $\delta=X(t) \tilde{\delta}(\beta, B)$. Now the holders are active and share the cost of planting and protection among themselves, hence an active option holder will spend $\left(C_{0} / N\right)\left(x-x_{0}\right)$ for initial planting, and $(1 / N) \int_{0}^{1} \tilde{\delta}(\beta, B) X(t) \mathrm{d} t$ for the protection during the time interval $[0,1]$.

Assuming linear utility function (for simplicity), the optimal action is determined by maximization of:

$$
\mathrm{E}\left[\int_{0}^{1}(X(s) \wedge k) \mathrm{d} s-C-\frac{C_{0}}{N}\left(x-x_{0}\right)-\frac{1}{N} \int_{0}^{1} \tilde{\delta}(\beta, B) X(s) \mathrm{d} s\right]
$$

with respect to $B$ and $x, C$ being the price of the option. The dynamic of the process is:

$$
\mathrm{d} X(t)=2 \sqrt{X(t)} \mathrm{d} W(t)+2 B X(t) \mathrm{d} t
$$

with

$$
X(0)=x .
$$

We chose linear utility because in this case we can solve the problem with the relatively easy use of numerical methods.

Of course, talking about awarding for planting trees we refer to undeveloped countries. It is well known that rural communities in undeveloped countries have a hierarchical structure dominated by powerful individuals. Some authors, for example Oates (1999), identify this as a failure of a community based approach in the past. Precisely, this hierarchical structure can help our purpose, and these powerful individuals could act as natural agents.

To start the project, we could give this options free from any charges (of course in reduced range). We will try to apply this approach in Mexico and this could give us better idea about models, parameters, and costs.

Let $\Sigma$ be a fund destinated to reforestation in given region. Now, we can sell $N=\Sigma /(k-C)$ options. Apparently, $N$ does not depend on the model. But $C$ must make the business worthy, so it depends on the chosen model. This is the first move in the game between reforestation and deforestation. Second move in the game would be selling put options, "bad options", with the payment

$$
\int_{0}^{1}[k-X(s)]_{+} \mathrm{d} s
$$


The price of this option should be high, so, to price it, we could apply the original model with parameters $\left(x_{0}, \beta\right)$ or even charge more. This will be the object of another study. We finish this section with the comment that environmentalists should buy bad option and do nothing. This would increase the fund.

\section{Comments about Financial Markets on Environment}

We are aiming toward the creation of markets out of environment, which is why we have to answer the following question: are there any opposite interests that help to create the market? Referring to this question and taking once again reforestation as an example, we can clearly identify opposing views. On the one hand, reforestation is desired by:

1. General public

2. Tourism, although excessive environmental tourism can be harmful as shown by the Galapagos example.

3. Lumber industry with long term vision.

4. Benefits of "analog forestry".

On the other hand, it is clear that not everyone embraces it, such as:

1. Cattle ranches and milk industries. (Sometimes these industries give cattle to small farmers for free).

2. Myopic lumber industries.

Some kind of opposite interest can be found in any environmental topic. Assume, now, that we have the market. In this case, it makes sense to talk about the value of $X(t)$ itself, even of buying $X(t)$. Of course, if work $\delta$ is implied, it would act as change from $r \rightarrow r+\delta$ in risk neutral valuation. The natural question is: can we solve the classical Merton problem of optimal investment if, instead of geometric Brownian motion, the underlying asset is $X(t)$ ? The answer is yes and we will show how to do it. Who could be the emisor of environmental options? In the worldwide scale, it should be done by United Nations dependencies. In the case of "AAA confidence" toward a given government, this government could be in charge of the management of resources and issue options.

\section{Merton's Problem}

We will give here a sketch of the solution. Assume that we can invest a part of our wealth $X$ in the risky asset with the dynamic:

$$
\mathrm{d} X(t)=2 \sqrt{X(t)} \mathrm{d} W(t)+2 \beta X(t) \mathrm{d} t
$$

and the risk free one with the rate $r$. In the first part, $r$ is equal to zero. We want to find the dynamical optimal portfolio that maximizes the utility of the final wealth.

$$
\mathrm{E}\left[u\left(Y_{1}^{\pi}\right)\right]
$$


where

$$
u(y)=\frac{y^{1-\alpha}}{1-\alpha}
$$

$\alpha>0$, and $\alpha \neq 1$. In our case, the state price density is the process $H$ defined as the solution of:

$$
\begin{gathered}
\mathrm{d} H_{t}=-H_{t}\left(r \mathrm{~d} t+\theta_{t} \mathrm{~d} W_{t}\right)=-H_{t}\left(r \mathrm{~d} t+\theta \sqrt{X_{t}} \mathrm{~d} W_{t}\right), H_{0}=1, \\
\theta_{t}=\frac{2 \beta-r}{2} \sqrt{X_{t}}=\theta \sqrt{X_{t}},
\end{gathered}
$$

is equal to

$$
H_{t}=e^{-r t} \exp \left\{-\int_{0}^{t} \theta \sqrt{X_{s}} \mathrm{~d} W_{s}-\frac{1}{2} \int_{0}^{t} \theta^{2} X_{s} \mathrm{~d} s\right\} .
$$

It is well known that the terminal optimal wealth is

$$
Y_{T}^{*}=\left(u^{\prime}\right)^{-1}\left(\nu H_{T}\right)
$$

where $\nu$ is a Lagrange multiplier which satisfies $\mathrm{E}\left(H_{T} Y_{T}^{*}\right)=x$, and the current optimal wealth is

$$
Y_{t}^{*} H_{t}=\mathrm{E}\left(Y_{T}^{*} H_{T} \mid \mathcal{F}_{t}\right)
$$

The optimal portfolio is obtained from a predictable representation theorem.

For a CRRA (constant relative risk aversion) utility function, a closed form solution for the optimal wealth can be found by an inversion of Pitman and Yor formula ( $c f$. Revuz and Yor 1998, p. 444), we drop the drift first using Girsanov theorem, and so

$$
Y_{t}^{*} H_{t}=\nu^{\gamma} \mathrm{E}\left(H_{T}^{\gamma+1} \mid \mathcal{F}_{t}\right),
$$

where $\gamma=-1 / \alpha$. The closed form solution for $H$ leads to

$$
\begin{aligned}
H_{T} & =H_{t} e^{-r(T-t)} \exp \left\{-\int_{t}^{T} \theta \sqrt{X_{s}} \mathrm{~d} W_{s}-\frac{1}{2} \int_{t}^{T} \theta^{2} X_{s} \mathrm{~d} s\right\} \\
& =H_{t} e^{-r(T-t)} \exp \left\{-\int_{t}^{T} \frac{\theta}{2} \mathrm{~d} X_{s}-\beta \theta \int_{t}^{T} X_{s} \mathrm{~d} s-\frac{1}{2} \int_{t}^{T} \theta^{2} X_{s} \mathrm{~d} s\right\} \\
& =H_{t} e^{-r(T-t)} \exp \left\{-\frac{\theta}{2}\left(X_{T}-X_{t}\right)-\left(\beta \theta+\frac{1}{2} \theta^{2}\right) \int_{t}^{T} X_{s} \mathrm{~d} s\right\} .
\end{aligned}
$$

Therefore, using that the pair $(X, H)$ is Markovian, there exists $\varphi$ such that

$$
\mathrm{E}\left(H_{T}^{\gamma+1} \mid \mathcal{F}_{t}\right)=H_{t}^{\gamma+1} e^{-(\gamma+1) r(T-t)} \varphi\left(t, X_{t}\right)
$$


where

$$
\varphi\left(t, X_{t}\right)=\mathrm{E}\left[e^{1+\gamma}\left(-\frac{\theta}{2}\left(X_{T}-X_{t}\right)-\beta \theta \int_{t}^{T} X_{s} \mathrm{~d} s-\frac{1}{2} \int_{t}^{T} \theta^{2} X_{s} \mathrm{~d} s\right) \mid \mathcal{F}_{t}\right]
$$

which can be calculated easily by Pitman and Yor formula. Now, the optimal wealth follows

$$
Y_{t}^{*}=\nu^{\gamma} H_{t}^{\gamma} \varphi\left(t, X_{t}\right),
$$

where

$$
H_{t}=e^{-r t} \exp \left\{-\frac{\theta}{2}\left(X_{t}-X_{0}\right)-\left(\beta \theta+\frac{1}{2} \theta^{2}\right) \int_{0}^{t} X_{s} \mathrm{~d} s\right\}
$$

so the problem can be solved explicitely.

\section{Further Valuation Topics}

In this section, we show how to solve valuation problems related to Section 3 . We do not have a market, so valuation means in the "real world". Unfortunately, we can only give solutions in terms of the Laplace transform. We are interested in calculations of:

(i). $\mathrm{E}_{x}\left[\int_{0}^{1}\left(X_{s} \wedge k\right) \mathrm{d} s I\left\{\inf _{0 \leq u \leq 1} X(s) \geq l\right\}\right]$

(ii). $\mathrm{E}_{x}\left[e^{-\lambda \int_{0}^{1} X(s) \mathrm{d} s} I\left\{\inf _{0 \leq u \leq 1} X(s) \geq l\right\}\right]$.

We start with a motivation for $(i)$ and (ii). In expression $(i)$, the term inside the expectations is clearly a better kind (in our case) of awards. Regarding (ii), it can be interpreted as the price of a bond if there is no "default". On the other hand, perhaps a better way of awarding could be paying for positive action, so our barrier option in $(i)$ should activate if $X(s)$ is large (the best way of awarding could be a kind of double barrier option). Ánalytically, it leads to the same kind of calculation. For calculation of $(i)$ and $(i i)$, we assume known the density $f_{x, \tau_{l}}$, where $X(0)=x$, and $\tau_{l}$ hitting time of the barrier $l$.

The problem of finding this density numerically could be of general interest, and we will try to solve it in another study. Because the dimension of the process is zero, the Laplace transform of hitting time of zero is

$$
\varphi_{x}(\alpha)=\alpha \int_{0}^{\infty} e^{-\alpha t} \exp \left\{\frac{-x \beta}{1-e^{-2 \beta t}}\right\} \mathrm{d} t
$$

Here, the integrand factor

$$
\exp \left\{\frac{-x \beta}{1-e^{-2 \beta t}}\right\}
$$


is the probability that $X(t)=0,(c f$. Revuz and Yor, 1998), and because zero is absorbing barrier this is also $P(\tau \leq t)$. Now, if $l<x$, then

$$
\varphi_{\tau_{l}}(\alpha)=\frac{\varphi_{\alpha}(x)}{\varphi_{\alpha}(l)}
$$

where $\varphi$ is the bounded decreasing solution of

$$
2 x \varphi^{\prime \prime}+2 \beta x \varphi^{\prime}=\alpha \varphi .
$$

Another solution for the Laplace transform is presented by Going and Yor (1999).

The knowledge of (ii) could lead to the knowledge of the joint law of $\int_{0}^{t} X(s) \mathrm{d} s$ and $\tau_{l}$. Here, we will use the parametrization:

$$
\mathrm{d} X(t)=2 \sqrt{X(t)} \mathrm{d} W(t)-2 \beta X(t) \mathrm{d} t, X(0)=x .
$$

Using Girsanov theorem, we have:

$$
\mathrm{E}_{x}\left[e^{-\lambda \int_{0}^{1} X(s) \mathrm{d} s} I\{\inf X(s) \geq l\}\right]=e^{A x} \mathrm{E}_{x}^{Q}\left[e^{-A X(1)} I\{\inf X(s) \geq l\}\right],
$$

where under $Q$

$$
\mathrm{d} X(t)=2 \sqrt{X(t)} \mathrm{d} W(t)+2 \sqrt{\beta^{2}+2 \lambda} X(t) \mathrm{d} t,
$$

and

$$
A=\frac{\beta+\sqrt{\beta^{2}+2 \lambda}}{2} .
$$

So if we know the density $f_{x, \tau_{l}}$, then

$$
\mathrm{E}_{x}^{Q}\left[e^{-A X(1)} I\{\inf X(s)=l\}\right]=\int_{0}^{1} \mathrm{E}^{Q}\left[e^{-A X(1)} \mid X_{\tau}=l\right] f_{x, \tau_{l}}(u) \mathrm{d} u .
$$

Of course, the term $E^{Q}(\cdot)$ is explicit by Pitman and Yor formula jointly with Girsanov theorem.

In order to calculate $(i)$, notice first that

$$
\begin{aligned}
\mathrm{E}_{x} & \int_{0}^{1}(X(s) \wedge k) \mathrm{d} s I\left\{\tau_{l} \geq 1\right\}=\int_{0}^{1} \mathrm{E}_{x}(X(s) \wedge k) \mathrm{d} s \\
& -\int_{0}^{1} \mathrm{E}_{x}\left[(X(s) \wedge k) I\left\{\tau_{l}<s\right\}\right] \mathrm{d} s-\int_{0}^{1} \mathrm{E}_{x}\left[(X(s) \wedge k) I\left\{\tau_{l} \in(s, 1)\right\}\right] \mathrm{d} s \\
& =\int_{0}^{1} \mathrm{E}_{x}(X(s) \wedge k) \mathrm{d} s-\int_{0}^{1} \int_{0}^{s} \mathrm{E}_{l}\left[(X(s) \wedge k) \mid \tau_{l}=u\right] f_{x, \tau_{l}}(u) \mathrm{d} u \mathrm{~d} s \\
& +\int_{0}^{1} \mathrm{E}_{x}\left[(X(s) \wedge k) \mathrm{E}\left(I\left\{\tau_{l} \in(s, 1)\right\} \mid \mathcal{F}_{s}\right)\right] \mathrm{d} s .
\end{aligned}
$$


Here, $\mathcal{F}$ is the filtration of $X(s)$. Therefore, knowing the law of $\tau_{l}$, we can get the explicit solution.

Another kind of paying premium can be obtained by modelling positive action as "anti-default". It means that there is no payment if there is no antidefault, which is modelled as default (occurs if $\sup _{0 \leq u \leq 1} X(u) \geq l^{*}$ ). Of course, it is a structural approach. It means that default is measurable in the filtration of the asset. There is also a natural way to introduce the intensity of the anti-default. This approach will lead to explicit solutions.

Define $\tau=\inf \left\{t: \int_{0}^{t} \lambda X(s) \mathrm{d} s=\Theta\right\}$ where $\Theta$ is an exponential random variable with parameter 1 independent of the process. Now we have:

\section{Proposition 2.}

For all $s \leq t$, it follows:

$$
\operatorname{Pr}\left(\tau>s \mid \mathcal{F}_{t}\right)=\exp \left\{-\int_{0}^{s} X(u) \mathrm{d} u\right\}
$$

Proof:

We have $\{\tau>s\}=\left\{\Theta>\int_{0}^{s} X(u) \mathrm{d} u\right\}$. From the independence and the $\mathcal{F}_{t}$ measurability of $\int_{0}^{t} X(u) \mathrm{d} u$

$$
\operatorname{Pr}\left(\tau>s \mid \mathcal{F}_{t}\right)=\operatorname{Pr}\left(\int_{0}^{s} X(u) \mathrm{d} u<\Theta \mid \mathcal{F}_{t}\right)=e^{-\int_{0}^{s} X(u) \mathrm{d} u}
$$

Now, if $\mathcal{G}_{t}=\mathcal{H}_{t} \vee \mathcal{F}_{t}$, where $\mathcal{H}_{t}$ is information about default (for details, $c f$. Elliott et al. 2000)

$$
\mathrm{E}\left(I_{T<\tau} \mid \mathcal{G}_{t}\right)=I_{t<\tau} \frac{\mathrm{E}\left(I(\tau>T) \mid \mathcal{F}_{t}\right)}{\mathrm{E}\left(I(\tau>t) \mid \mathcal{F}_{t}\right)}=I_{t<\tau} \mathrm{E}\left\{e^{-\int_{t}^{T} X(u) \mathrm{d} u} \mid \mathcal{F}_{t}\right\} .
$$

We can also obtain an intensity (generalized) based approach using time reversal technique. Set

$$
\begin{gathered}
\mathrm{d} Y(t)=2 \sqrt{Y(t)} \mathrm{d} W(t), Y(0)=x, \\
\mathrm{~d} \tilde{Y}(t)=2 \sqrt{\tilde{Y}(t)} \mathrm{d} W(t)+4 \mathrm{~d} t, \tilde{Y}(0)=0,
\end{gathered}
$$

and

$$
\tilde{V}(t)=Y\left(\tau_{t}\right)
$$

where

$$
\tau_{t}=\frac{1-e^{-2 \beta t}}{2 \beta} .
$$

Note that $e^{2 \beta t} Y\left(\tau_{t}\right)$ is our model from section 2 . 
Define default as a hitting time $\delta$ of zero by the process $Y(t)$. This default time does not admit the intensity ( $c f$. Elliott et al. 2000). Using time reversal theorem, we have:

$$
\operatorname{Pr}(\delta<t)=\operatorname{Pr}\left(\gamma_{x}<t\right),
$$

where $\gamma_{x}$ is the last $x$ of the process $\tilde{Y}$. Let $H_{t}$ be the filtration of "default" $\gamma_{x}$, and $\mathcal{G}_{t}=H_{t} \vee \mathcal{F}_{t}^{\bar{Y}}$. The process $\tilde{Y}$ is a transient diffusion, and therefore the default $\gamma_{x}$ has a compensator $\wedge_{t}$ in the filtration $\mathcal{F}$. We have $\wedge_{t}=(x / 2) L_{t}^{x / 2}$, where $L$ is the local time of the local martingale $1 / \tilde{Y}(t)$. We choose the bicontinuous modification of the local time. Standard calculations lead to the compensator of default in $\mathcal{F}$ filtration as $(1 / 2 x) L_{t}^{x}(\tilde{Y})$. We also have:

$$
\operatorname{Pr}\left(\gamma_{x}>t \mid \mathcal{F}_{t}^{\bar{Y}}\right)=e^{-\Gamma_{t}}=\frac{x}{\tilde{Y}(t)} \wedge 1
$$

This formula allows the calculation of defaultable claims as in Elliott et al. (2000). Considering the hitting time of zero by the process $X(t)$, we have:

$$
\operatorname{Pr}\left(\delta^{X}<t\right)=\operatorname{Pr}\left(\delta \leq \tau_{t}\right)=\operatorname{Pr}\left(\gamma_{x}^{\tilde{Y}}<\tau_{t}\right) .
$$

Therefore,

$$
e^{-\Gamma_{t}^{\tilde{Y}}}=\operatorname{Pr}\left(\gamma_{x}>\tau_{t} \mid \mathcal{F}_{\tau_{t}}^{\tilde{Y}}\right)=\frac{x}{\tilde{V}\left(\tau_{\tilde{t}}\right)} \wedge 1
$$

\section{Conclusions}

We have shown that the financial approach, based on branching phenomena, is practically the only one able to stop and invert the environmental degradation. We focused on deforestation and showed how to produce effectively reforestation. In the game of reforestation versus deforestation, we have solve the optimization problem of the first move needed to start the project. Needless to say, further research is required to obtain more and better results regarding environment and finance.

\section{References}

Crabbe, P., A. J. Holland, L. Ryszkowski, and L. Westra (2000). Implementing Ecological Integrity. Kluwer Academic Publishers.

Elliott, R., M. Jeanblanc, and M. Yor (2000). On Models of Default Risk. Mathematical Finance, 10, pp. 179-195.

Ethier, S., and T. Kurtz (1986). Markov Processes Characterization and Convergence. Wiley.

Going, A., and M. Yor (1999). A Survey and Some Generalizations of Bessel Processes. Preprint.

Merton, R, and Z. Bodie (1995). A Conceptual Framework for Analyzing the Financial Environment. In the Global Financial System: A Functional Perspective. Harvard Business School Press.

Oates, J. (1999). Myth and Reality in the Rain Forest. University of California Press.

Revuz, D., and M. Yor (1998). Continuous Martingales and Brownian Motion. SpringerVerlag.

Schmidli, H. P. (1992). A General Insurance Risk Models. Ph. D. Thesis, TH Zurich.

Davis, M. H. A. (1984). Piecewise Deterministic Markov Processes. J. R. Statist. Soc. B., 46, pp. 353-388. 